\title{
Comparative Analysis of Rate of Convergence of Agarwal et al., Noor and SP iterative schemes for Complex Space
}

\author{
Renu Chugh, Vivek Kumar ,Ombir Dahiya \\ Department of Mathematics \\ Maharshi Dayanand University, \\ Rohtak, India
}

\begin{abstract}
In this paper, we analyze the rate of convergence of three iterative schemes namely-Agarwal et al., Noor and SP iterative schemes for complex space by using Matlab programmes. The results obtained are extensions of some recent results of Rana, Dimri and Tomar[1].
\end{abstract}

\section{General Terms}

Computational Mathematics

\section{Keywords}

Agarwal et al. iterative scheme, Noor iterative scheme, SP iterative scheme

\section{INTRODUCTION}

Although fixed point iterative schemes are used in solving problems of industrial and applied mathematics still there is no systematic study of numerical aspects of these iterative schemes. In computational mathematics, it is important to compare the iterative schemes with regard to their rate of convergence. By using computer programs, perhaps for the first time, B. E. Rhoades [2] illustrated the difference in convergence rate of Mann and Ishikawa iterative procedures for increasing and deceasing functions through examples. S. L. Singh[3] extended the work of Rhoades. In [4,5] Berinde showed that Picard iteration converges faster than Mann iteration for quasi-contractive operators.

Recently, Nawab Hussian et al. [6] provide an example of a quasi-contractive operator for which the iterative scheme due to Agarwal et al. is faster than Mann and Ishikawa iterative schemes. By providing examples, W. Phuengrattana and S. Suantai[7] showed that SP iterative scheme converges faster than Mann, Ishikawa and Noor iterative for nondecreasing and continuous functions on real line intervals. Recently, Rana, Dimri and Tomar[1] showed that Ishikawa iterative scheme converges faster than Mann iterative scheme while Picard iterative scheme converges faster than both in complex space.

\section{PRELIMINARIES}

Let $(X, d)$ be a complete metric space and $T: X \rightarrow X$ a selfmap of $X$. Suppose that $F(T)=\{p \in X, T p=p\}$ is the set of fixed points of $T$. There are several iterative processes in the literature for which the fixed points of operators have been approximated over the years by various authors. In a complete metric space, the Picard iterative process $\left\{x_{n}\right\}_{n=0}^{\infty}$ defined by

$$
x_{n+1}=T x_{n} n=0,1, \ldots
$$

is used to approximate the fixed points of mappings satisfying the following Banach's contraction condition:

$$
d(T x, T y) \leq \alpha d(x, y)
$$

for all $x, y \in X$ and $\alpha \in[0,1)$.

In 1953, W. R. Mann defined the Mann iteration [8] as

$$
x_{n+1}=\left(1-\alpha_{n}\right) x_{n}+\alpha_{n} T x_{n}
$$

where $\left\{\alpha_{\mathrm{n}}\right\}$ is a sequences of positive numbers in $[0,1]$. In 1974, S. Ishikawa defined the Ishikawa iteration [9] as

$$
\begin{aligned}
x_{n+1} & =\left(1-\alpha_{n}\right) x_{n}+\alpha_{n} T y_{n} \\
y_{n} & =\left(1-\beta_{n}\right) x_{n}+\beta_{n} T x_{n},
\end{aligned}
$$

where $\left\{\alpha_{\mathrm{n}}\right\}$ and $\left\{\beta_{\mathrm{n}}\right\}$ are sequences of positive numbers in $[0,1]$.

In 2007, Agarwal et al. defined the Agarwal et al. iterative scheme [10] as

$$
\begin{aligned}
s_{n+1} & =\left(1-\alpha_{n}\right) T s_{n}+\alpha_{n} T t_{n} \\
t_{n} & =\left(1-\beta_{n}\right) s_{n}+\beta_{n} T s_{n},
\end{aligned}
$$

where $\left\{\alpha_{\mathrm{n}}\right\}$ and $\left\{\beta_{\mathrm{n}}\right\}$ are sequences of positive numbers in $[0,1]$.

In 2000, M. A. Noor defined the three step Noor iteration $[11]$ as

$$
\begin{gathered}
x_{n+1}=\left(1-\alpha_{n}\right) x_{n}+\alpha_{n} T y_{n} \\
y_{n}=\left(1-\beta_{n}\right) x_{n}+\beta_{n} T z_{n} \\
z_{n}=\left(1-\gamma_{n}\right) x_{n}+\gamma_{n} T x_{n},
\end{gathered}
$$

where $\left\{\alpha_{\mathrm{n}}\right\},\left\{\beta_{\mathrm{n}}\right\}$ and $\left\{\gamma_{\mathrm{n}}\right\}$ are sequences of positive numbers in $[0,1]$.

Phuengrattana and Suantai defined the SP iteration [7] as

$$
\begin{gathered}
x_{n+1}=\left(1-\alpha_{n}\right) y_{n}+\alpha_{n} T y_{n} \\
y_{n}=\left(1-\beta_{n}\right) z_{n}+\beta_{n} T z_{n} \\
z_{n}=\left(1-\gamma_{n}\right) x_{n}+\gamma_{n} T x_{n},
\end{gathered}
$$

where $\left\{\alpha_{\mathrm{n}}\right\},\left\{\beta_{\mathrm{n}}\right\}$ and $\left\{\gamma_{\mathrm{n}}\right\}$ are sequences of positive numbers in $[0,1]$.

\section{Remarks}

1. If $\gamma_{\mathrm{n}}=0$, then Noor iteration (2.6) reduces to the Ishikawa iteration (2.4). 
2. If $\beta_{\mathrm{n}}=\gamma_{\mathrm{n}}=0$, then Noor iteration (2.6) reduces to the Mann iteration (2.3).

3. If $\beta_{\mathrm{n}}=\gamma_{\mathrm{n}}=0$, then SP iteration (2.7) reduces to the Mann iteration (2.3).

In this paper, we take $\alpha_{\mathrm{n}}=s, \beta_{\mathrm{n}}=s^{\prime}, \gamma_{\mathrm{n}}=s^{\prime \prime}$ and derive the fixed points of the following polynomial functions :

Quadratic functions $=z^{2}+c$

Cubic functions $=z^{3}+c$

Biquadratic functions $=z^{4}+c$

Recently, Rana, Dimri and Tomar[1] draw a comparative analysis of Picard, Mann and Ishikawa iterative schemes by starting with $z=(0,0)$ and $c=0.1$ in complex space.

In this paper, we will continue the comparative study in complex space by taking same $z$ and $c$, for Agarwal et al., Noor and SP iterative schemes and hence extend the results of Rana, Dimri and Tomar[1] .

\section{EXPERIMENTS}

\subsection{Fixed points of quadratic polynomial}

Table 1 (Agarwal et al. iteration ) $s=0.6, s^{\prime}=0.1$

\begin{tabular}{|c|c|c|c|}
\hline $\begin{array}{c}\text { Number } \\
\text { of } \\
\text { iterations }\end{array}$ & $x_{n}$ & $\begin{array}{c}\text { Number } \\
\text { of } \\
\text { iterations }\end{array}$ & $x_{n}$ \\
\hline 1 & 0.10006 & 6 & 0.112696 \\
\hline 2 & 0.110132 & 7 & 0.112701 \\
\hline 3 & 0.112155 & 8 & 0.112701 \\
\hline 4 & 0.112585 & 9 & $\mathbf{0 . 1 1 2 7 0 2}$ \\
\hline 5 & 0.112677 & 10 & $\mathbf{0 . 1 1 2 7 0 2}$ \\
\hline
\end{tabular}

Figure 1 (Agarwal et al. iteration )

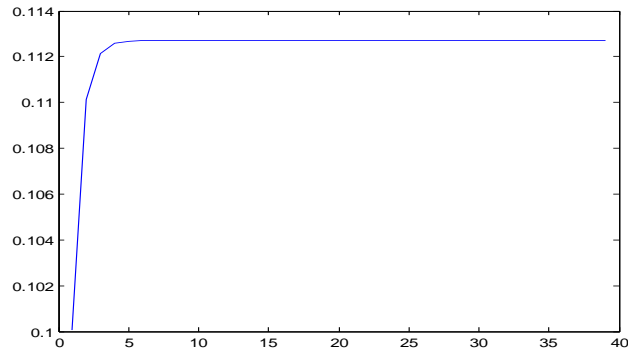

Table 2 (Noor iteration) $s=\mathbf{0 . 6}, s^{\prime}=s^{\prime \prime}=\mathbf{0 . 1}$

\begin{tabular}{|c|c|c|c|}
\hline $\begin{array}{l}\text { Number } \\
\text { of } \\
\text { iterations }\end{array}$ & $x_{n}$ & $\begin{array}{c}\text { Number } \\
\text { of } \\
\text { iterations }\end{array}$ & $x_{n}$ \\
\hline 1 & 0.06006 & 12 & 0.112663 \\
\hline 2 & 0.086518 & 13 & 0.112681 \\
\hline 3 & 0.099323 & 14 & 0.112691 \\
\hline 4 & 0.105777 & 15 & 0.112696 \\
\hline 5 & 0.109094 & 16 & 0.112699 \\
\hline 6 & 0.110816 & 17 & 0.1127 \\
\hline 7 & 0.111715 & 18 & 0.112701 \\
\hline 8 & 0.112184 & 19 & 0.112701 \\
\hline 9 & 0.11243 & 20 & 0.112701 \\
\hline 10 & 0.112559 & 21 & $\mathbf{0 . 1 1 2 7 0 2}$ \\
\hline 11 & 0.112627 & 22 & $\mathbf{0 . 1 1 2 7 0 2}$ \\
\hline
\end{tabular}

Figure 2 ( Noor iteration)

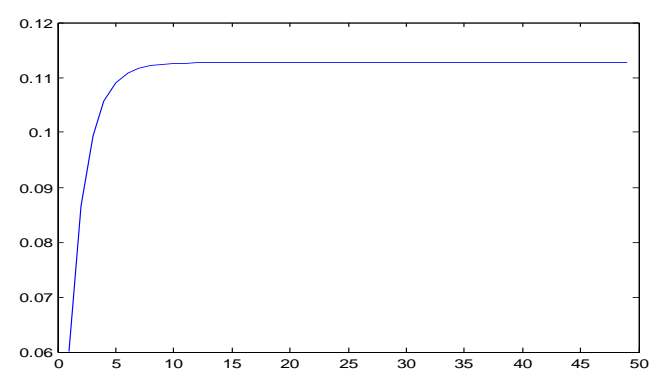

Table 3 (SP iteration ) $s=0.6, s^{\prime}=s^{\prime \prime}=0.1$

\begin{tabular}{|c|c|c|c|}
\hline $\begin{array}{c}\text { Number } \\
\text { of } \\
\text { iterations }\end{array}$ & $x_{n}$ & $\begin{array}{c}\text { Number } \\
\text { of } \\
\text { iterations }\end{array}$ & $x_{n}$ \\
\hline 1 & 0.067821 & 10 & 0.112667 \\
\hline 2 & 0.093306 & 11 & 0.112686 \\
\hline 3 & 0.104064 & 12 & 0.112695 \\
\hline 4 & 0.108806 & 13 & 0.112698 \\
\hline 5 & 0.110935 & 14 & 0.1127 \\
\hline 6 & 0.111899 & 15 & 0.112701 \\
\hline 7 & 0.112336 & 16 & 0.112701 \\
\hline 8 & 0.112535 & 17 & $\mathbf{0 . 1 1 2 7 0 2}$ \\
\hline 9 & 0.112626 & 18 & $\mathbf{0 . 1 1 2 7 0 2}$ \\
\hline
\end{tabular}

Figure 3 (SP iteration)

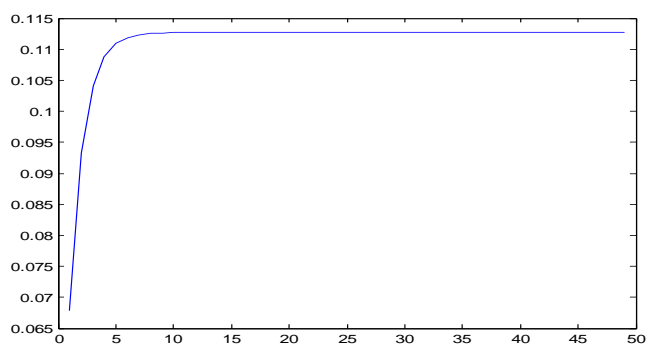

Table 4 (Agarwal et al. iteration ) $s=0.6, s^{\prime}=0.3$

\begin{tabular}{|c|c|c|c|}
\hline $\begin{array}{c}\text { Number } \\
\text { of } \\
\text { iterations }\end{array}$ & $x_{n}$ & $\begin{array}{c}\text { Number } \\
\text { of } \\
\text { iterations }\end{array}$ & $x_{n}$ \\
\hline 1 & 0.10054 & 6 & 0.112699 \\
\hline 2 & 0.11046 & 7 & 0.112701 \\
\hline 3 & 0.112271 & 8 & $\mathbf{0 . 1 1 2 7 0 2}$ \\
\hline 4 & 0.112618 & 9 & $\mathbf{0 . 1 1 2 7 0 2}$ \\
\hline 5 & 0.112685 & 10 & $\mathbf{0 . 1 1 2 7 0 2}$ \\
\hline
\end{tabular}


Figure 4 (Agarwal et al. iteration )

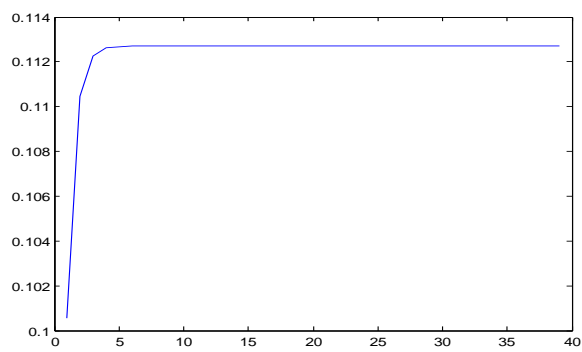

Table 5 (Noor iteration ) $s=\mathbf{0 . 6}, s^{\prime}=0.3, s^{\prime \prime}=0.1$

\begin{tabular}{|c|c|c|c|}
\hline $\begin{array}{c}\text { Number } \\
\text { of } \\
\text { iterations }\end{array}$ & $x_{n}$ & $\begin{array}{c}\text { Number } \\
\text { of } \\
\text { iterations }\end{array}$ & $x_{n}$ \\
\hline 1 & 0.060541 & 12 & 0.112676 \\
\hline 2 & 0.08747 & 13 & 0.112689 \\
\hline 3 & 0.100248 & 14 & 0.112695 \\
\hline 4 & 0.106495 & 15 & 0.112698 \\
\hline 5 & 0.109594 & 16 & 0.1127 \\
\hline 6 & 0.111142 & 17 & 0.112701 \\
\hline 7 & 0.111918 & 18 & 0.112701 \\
\hline 8 & 0.112308 & 19 & 0.112701 \\
\hline 9 & 0.112503 & 20 & $\mathbf{0 . 1 1 2 7 0 2}$ \\
\hline 10 & 0.112602 & 21 & $\mathbf{0 . 1 1 2 7 0 2}$ \\
\hline 11 & 0.112652 & 22 & $\mathbf{0 . 1 1 2 7 0 2}$ \\
\hline
\end{tabular}

Figure 5 (Noor iteration)

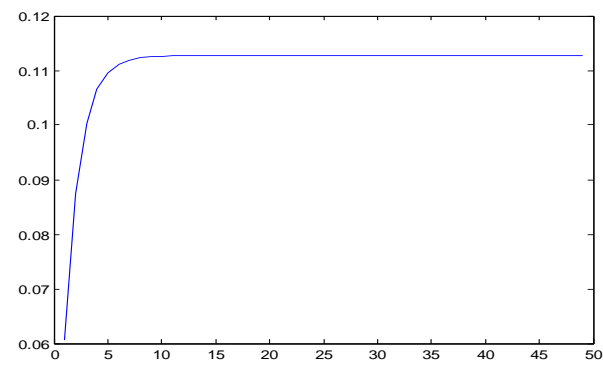

Table 6 (SP iteration) $s=0.6, s^{\prime}=0.3, s^{\prime \prime}=0.1$

\begin{tabular}{|c|c|c|c|}
\hline $\begin{array}{c}\text { Number } \\
\text { of } \\
\text { iterations }\end{array}$ & $x_{n}$ & $\begin{array}{c}\text { Number } \\
\text { of } \\
\text { iterations }\end{array}$ & $x_{n}$ \\
\hline 1 & 0.075635 & 9 & 0.112687 \\
\hline 2 & 0.099294 & 10 & 0.112696 \\
\hline 3 & 0.107705 & 11 & 0.1127 \\
\hline 4 & 0.11082 & 12 & 0.112701 \\
\hline 5 & 0.11199 & 13 & 0.112701 \\
\hline 6 & 0.112432 & 14 & $\mathbf{0 . 1 1 2 7 0 2}$ \\
\hline 7 & 0.1126 & 15 & $\mathbf{0 . 1 1 2 7 0 2}$ \\
\hline 8 & 0.112663 & 16 & $\mathbf{0 . 1 1 2 7 0 2}$ \\
\hline
\end{tabular}

Figure 6 ( SP iteration)

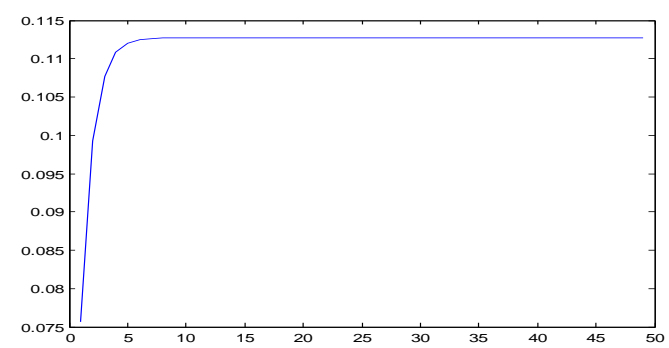

Table 7 (Agarwal et al. iteration ) $s=0.6, s^{\prime}=0.5$

\begin{tabular}{|c|c|c|c|}
\hline $\begin{array}{c}\text { Number } \\
\text { of } \\
\text { iterations }\end{array}$ & $x_{n}$ & $\begin{array}{c}\text { Number } \\
\text { of } \\
\text { iterations }\end{array}$ & $x_{n}$ \\
\hline 1 & 0.1015 & 6 & 0.1127 \\
\hline 2 & 0.11085 & 7 & 0.112701 \\
\hline 3 & 0.112384 & 8 & $\mathbf{0 . 1 1 2 7 0 2}$ \\
\hline 4 & 0.112647 & 9 & $\mathbf{0 . 1 1 2 7 0 2}$ \\
\hline 5 & 0.112692 & 10 & $\mathbf{0 . 1 1 2 7 0 2}$ \\
\hline
\end{tabular}

Figure 7 (Agarwal et al. iteration )

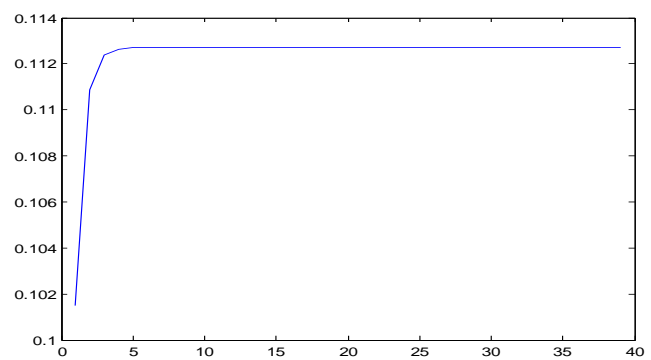

Table 8 (Noor iteration ) $s=0.6, s^{\prime}=0.5, s^{\prime \prime}=0.4$

\begin{tabular}{|c|c|c|c|}
\hline $\begin{array}{c}\text { Number } \\
\text { of } \\
\text { iterations }\end{array}$ & $x_{n}$ & $\begin{array}{c}\text { Number } \\
\text { of } \\
\text { iterations }\end{array}$ & $x_{n}$ \\
\hline 1 & 0.061548 & 12 & 0.112687 \\
\hline 2 & 0.088838 & 13 & 0.112695 \\
\hline 3 & 0.101424 & 14 & 0.112698 \\
\hline 4 & 0.107339 & 15 & 0.1127 \\
\hline 5 & 0.110145 & 16 & 0.112701 \\
\hline 6 & 0.111481 & 17 & 0.112701 \\
\hline 7 & 0.112118 & 18 & 0.112701 \\
\hline 8 & 0.112423 & 19 & $\mathbf{0 . 1 1 2 7 0 2}$ \\
\hline 9 & 0.112568 & 20 & $\mathbf{0 . 1 1 2 7 0 2}$ \\
\hline 10 & 0.112638 & 21 & $\mathbf{0 . 1 1 2 7 0 2}$ \\
\hline 11 & 0.112671 & 22 & $\mathbf{0 . 1 1 2 7 0 2}$ \\
\hline
\end{tabular}


Figure 8 (Noor iteration)

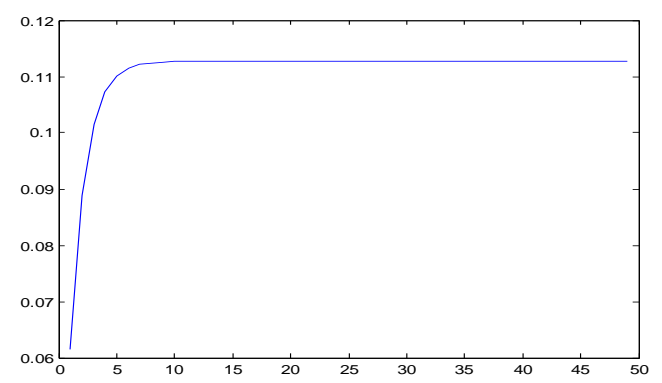

Table 9 (SP iteration) $s=0.6, s^{\prime}=0.5, s^{\prime \prime}=0.4$

\begin{tabular}{|c|c|c|l|}
\hline $\begin{array}{c}\text { Number } \\
\text { of } \\
\text { iterations }\end{array}$ & $x_{n}$ & $\begin{array}{c}\text { Number } \\
\text { of } \\
\text { iterations }\end{array}$ & $x_{n}$ \\
\hline 1 & 0.091328 & 6 & 0.11269 \\
\hline 2 & 0.108027 & 7 & 0.112699 \\
\hline 3 & 0.111652 & 8 & 0.112701 \\
\hline 4 & 0.112464 & 9 & $\mathbf{0 . 1 1 2 7 0 2}$ \\
\hline 5 & 0.112648 & 10 & $\mathbf{0 . 1 1 2 7 0 2}$ \\
\hline
\end{tabular}

Figure 9 (SP iteration )

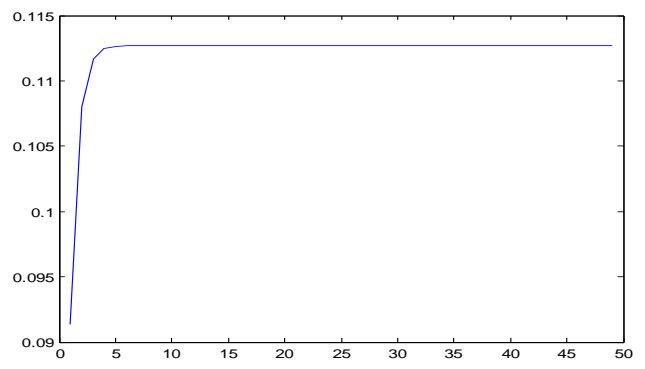

Table 10 (Agarwal et al. iteration ) $s=0.6, s^{\prime}=0.8$

\begin{tabular}{|l|l|l|l|}
\hline $\begin{array}{l}\text { Number } \\
\text { of } \\
\text { iterations }\end{array}$ & $x_{n}$ & $\begin{array}{l}\text { Number } \\
\text { of } \\
\text { iterations }\end{array}$ & $x_{n}$ \\
\hline 1 & 0.10384 & 5 & 0.112698 \\
\hline 2 & 0.111493 & 6 & 0.112701 \\
\hline 3 & 0.112531 & 7 & $\mathbf{0 . 1 1 2 7 0 2}$ \\
\hline 4 & 0.112678 & 8 & $\mathbf{0 . 1 1 2 7 0 2}$ \\
\hline
\end{tabular}

Figure 10 (Agarwal et al. iteration )

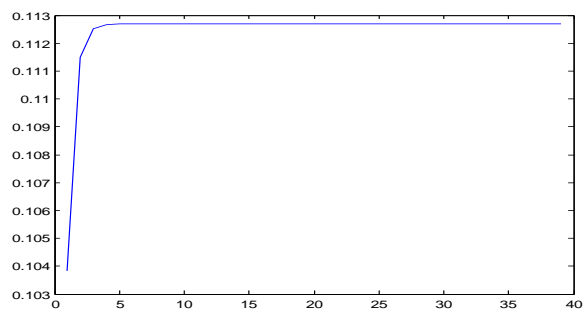

Table 11 (Noor iteration) $s=0.6, s^{\prime}=0.8, s^{\prime \prime}=.7$

\begin{tabular}{|c|l|c|c|}
\hline $\begin{array}{c}\text { Number } \\
\text { of } \\
\text { iterations }\end{array}$ & $x_{n}$ & $\begin{array}{c}\text { Number } \\
\text { of } \\
\text { iterations }\end{array}$ & $x_{n}$ \\
\hline 1 & 0.064226 & 12 & 0.112696 \\
\hline 2 & 0.091647 & 13 & 0.112699 \\
\hline 3 & 0.103513 & 14 & 0.112701 \\
\hline
\end{tabular}

\begin{tabular}{|c|l|l|l|}
\hline 4 & 0.108682 & 15 & 0.112701 \\
\hline 5 & 0.110942 & 16 & 0.112701 \\
\hline 6 & 0.111931 & 17 & $\mathbf{0 . 1 1 2 7 0 2}$ \\
\hline 7 & 0.112364 & 18 & $\mathbf{0 . 1 1 2 7 0 2}$ \\
\hline 8 & 0.112554 & 19 & $\mathbf{0 . 1 1 2 7 0 2}$ \\
\hline 9 & 0.112637 & 20 & $\mathbf{0 . 1 1 2 7 0 2}$ \\
\hline 10 & 0.112673 & 21 & $\mathbf{0 . 1 1 2 7 0 2}$ \\
\hline 11 & 0.112689 & 22 & $\mathbf{0 . 1 1 2 7 0 2}$ \\
\hline
\end{tabular}

Figure 11 (Noor iteration)

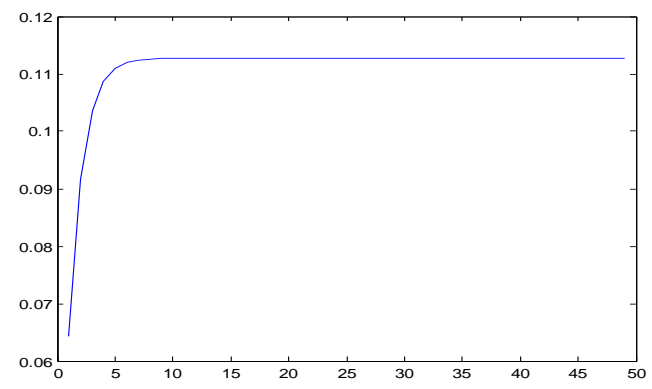

Table 12 (SP iteration) $s=\mathbf{0 . 6}, s^{\prime}=0.8, s^{\prime \prime}=0.7$

\begin{tabular}{|c|c|}
\hline Number of iterations & $x_{n}$ \\
\hline 1 & 0.104921 \\
\hline 2 & 0.111992 \\
\hline 3 & 0.112636 \\
\hline 4 & 0.112696 \\
\hline 5 & 0.112701 \\
\hline 6 & $\mathbf{0 . 1 1 2 7 0 2}$ \\
\hline 7 & $\mathbf{0 . 1 1 2 7 0 2}$ \\
\hline
\end{tabular}

Figure 12 (SP iteration)

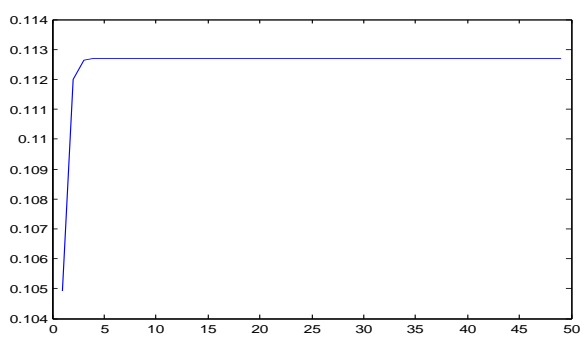

3.2 Fixed points of cubic polynomial

Table 13 (Agarwal et al. iteration ) $s=0.6, s=0.1$

\begin{tabular}{|c|c|}
\hline Number of iterations & $x_{n}$ \\
\hline 1 & 0.100001 \\
\hline 2 & 0.101002 \\
\hline 3 & 0.10103 \\
\hline 4 & $\mathbf{0 . 1 0 1 0 3 1}$ \\
\hline 5 & $\mathbf{0 . 1 0 1 0 3 1}$ \\
\hline 6 & $\mathbf{0 . 1 0 1 0 3 1}$ \\
\hline
\end{tabular}


Figure 13 (Agarwal et al. iteration )

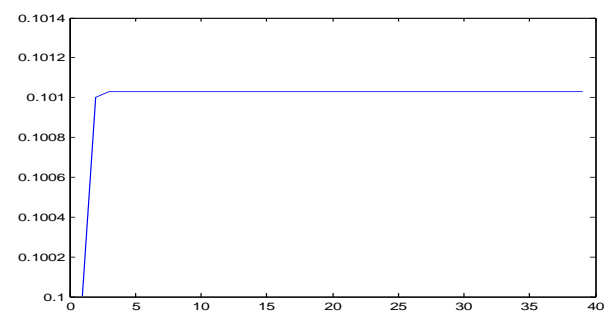

Table 14 (Noor iteration) $s=\mathbf{0 . 6}, s^{\prime}=s^{\prime \prime}=0.1$

\begin{tabular}{|c|c|c|c|}
\hline $\begin{array}{c}\text { Number } \\
\text { of } \\
\text { iterations } \\
1\end{array}$ & $x_{n}$ & $\begin{array}{c}\text { Number } \\
\text { of } \\
\text { iterations } \\
9\end{array}$ & $x_{n}$ \\
\hline 2 & 0.060001 & 0.100995 \\
\hline 3 & 0.094042 & 11 & 0.101016 \\
\hline 4 & 0.098127 & 12 & 0.101025 \\
\hline 5 & 0.099823 & 13 & 0.10103 \\
6 & 0.100528 & 14 & $\mathbf{0 . 1 0 1 0 3 1}$ \\
\hline 7 & 0.100822 & 15 & $\mathbf{0 . 1 0 1 0 3 1}$ \\
\hline 8 & 0.100944 & 16 & $\mathbf{0 . 1 0 1 0 3 1}$ \\
\hline
\end{tabular}

Figure 14 (Noor iteration)

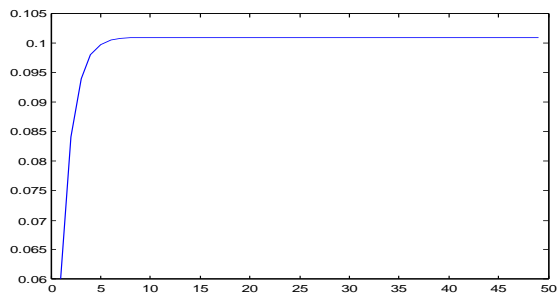

Table 15 (SP iteration) $s=0.6, s^{\prime}=s^{\prime \prime}=0.1$

\begin{tabular}{|c|c|c|c|}
\hline $\begin{array}{c}\text { Nu } \\
\text { mb } \\
\text { er } \\
\text { of } \\
\text { iter } \\
\text { ati } \\
\text { ons }\end{array}$ & $x_{n}$ & $\begin{array}{c}\text { Number } \\
\text { of } \\
\text { iterations }\end{array}$ & $x_{n}$ \\
\hline 1 & 0.067604 & 8 & 0.101014 \\
\hline 2 & 0.089771 & 9 & 0.101025 \\
\hline 3 & 0.097207 & 10 & 0.101029 \\
\hline 4 & 0.099728 & 11 & 0.101031 \\
\hline 5 & 0.100587 & 12 & 0.101031 \\
\hline 6 & 0.10088 & 13 & 0.101031 \\
\hline 7 & 0.10098 & 14 & 0.101031 \\
\hline
\end{tabular}

Figure 15 (SP iteration)

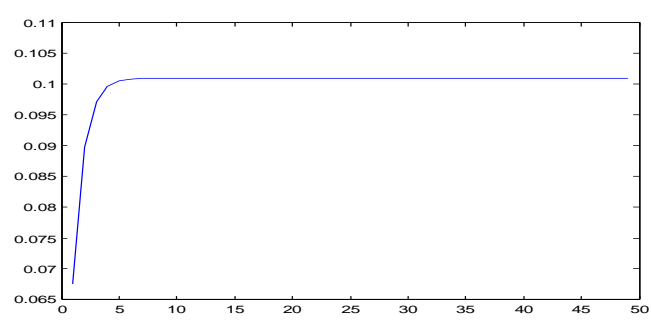

Table 16 (Agarwal et al. iteration ) $s=0.6, s^{\prime}=0.3$

\begin{tabular}{|c|c|}
\hline Number of iterations & $x_{n}$ \\
\hline 1 & 0.100016 \\
\hline 2 & 0.101006 \\
\hline 3 & $\mathbf{0 . 1 0 1 0 3 1}$ \\
\hline 4 & $\mathbf{0 . 1 0 1 0 3 1}$ \\
\hline 5 & $\mathbf{0 . 1 0 1 0 3 1}$ \\
\hline
\end{tabular}

Figure 16 (Agarwal et al. iteration )

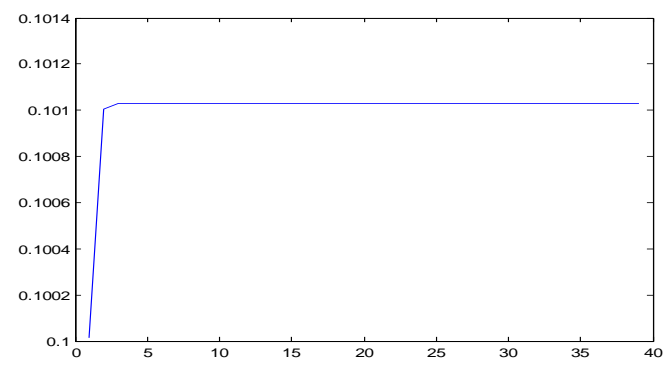

Table 17 (Noor iteration) $s=\mathbf{0 . 6}, s^{\prime}=0.3, s^{\prime \prime}=0.1$

\begin{tabular}{|c|c|c|c|}
\hline $\begin{array}{c}\text { Number } \\
\text { of } \\
\text { iterations }\end{array}$ & $x_{n}$ & $\begin{array}{c}\text { Number of } \\
\text { iterations }\end{array}$ & $x_{n}$ \\
\hline 1 & 0.060016 & 9 & 0.100997 \\
\hline 2 & 0.084231 & 10 & 0.101017 \\
\hline 3 & 0.094118 & 11 & 0.101025 \\
\hline 4 & 0.09818 & 12 & 0.101029 \\
5 & 0.099854 & 13 & 0.10103 \\
\hline 6 & 0.100545 & 14 & $\mathbf{0 . 1 0 1 0 3 1}$ \\
\hline 7 & 0.100831 & 15 & $\mathbf{0 . 1 0 1 0 3 1}$ \\
\hline 8 & 0.100948 & 16 & $\mathbf{0 . 1 0 1 0 3 1}$ \\
\hline
\end{tabular}

Figure 17 (Noor iteration)

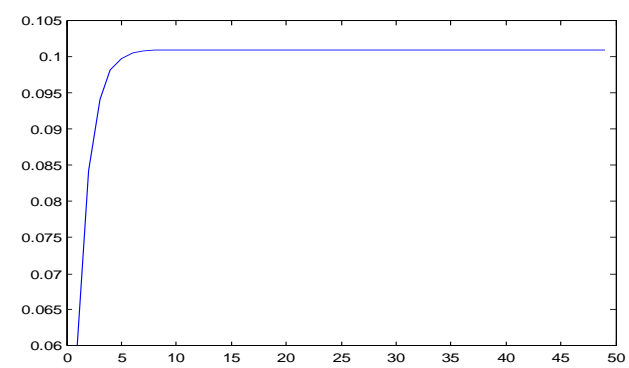

Table 18 ( SP iteration) $s=0.6, s^{\prime}=0.3, s^{\prime \prime}=0.1$

\begin{tabular}{|c|c|c|c|}
\hline $\begin{array}{c}\text { Number } \\
\text { of } \\
\text { iterations }\end{array}$ & $x_{n}$ & $\begin{array}{c}\text { Number } \\
\text { of } \\
\text { iterations }\end{array}$ & $x_{n}$ \\
\hline 1 & 0.074831 & 7 & 0.101022 \\
2 & 0.094084 & 8 & 0.101029 \\
3 & 0.099175 & 9 & $\mathbf{0 . 1 0 1 0 3 1}$ \\
\hline 4 & 0.100534 & 10 & $\mathbf{0 . 1 0 1 0 3 1}$ \\
\hline 5 & 0.100898 & 11 & $\mathbf{0 . 1 0 1 0 3 1}$ \\
\hline 6 & 0.100996 & 12 & $\mathbf{0 . 1 0 1 0 3 1}$ \\
\hline
\end{tabular}


Figure 18 (SP iteration)

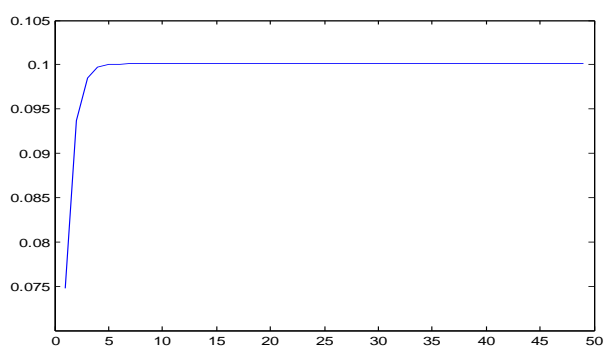

Table 19 (Agarwal et al. iteration ) $s=0.6, s^{\prime}=0.5$

\begin{tabular}{|c|c|}
\hline Number of iterations & $x_{n}$ \\
\hline 1 & 0.100075 \\
\hline 2 & 0.101011 \\
\hline 3 & $\mathbf{0 . 1 0 1 0 3 1}$ \\
\hline 4 & $\mathbf{0 . 1 0 1 0 3 1}$ \\
\hline 5 & $\mathbf{0 . 1 0 1 0 3 1}$ \\
\hline 6 & $\mathbf{0 . 1 0 1 0 3 1}$ \\
\hline
\end{tabular}

Figure 19 (Agarwal et al. iteration )

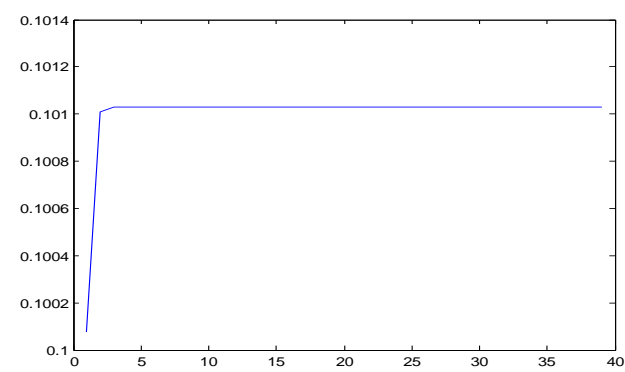

Table 20 (Noor iteration) $s=\mathbf{0 . 6}, s^{\prime}=0.5, s^{\prime \prime}=0.4$

\begin{tabular}{|c|l|c|c|}
\hline $\begin{array}{c}\text { Number } \\
\text { of } \\
\text { iterations }\end{array}$ & \multicolumn{1}{|c|}{$x_{n}$} & $\begin{array}{c}\text { Number } \\
\text { of } \\
\text { iterations }\end{array}$ & $x_{n}$ \\
\hline 1 & 0.060075 & 9 & 0.100999 \\
\hline 2 & 0.08434 & 10 & 0.101018 \\
\hline 3 & 0.094212 & 11 & 0.101026 \\
\hline 4 & 0.098242 & 12 & 0.101029 \\
\hline 5 & 0.09989 & 13 & 0.10103 \\
6 & 0.100564 & 14 & $\mathbf{0 . 1 0 1 0 3 1}$ \\
\hline 7 & 0.10084 & 15 & $\mathbf{0 . 1 0 1 0 3 1}$ \\
\hline 8 & 0.100953 & 16 & $\mathbf{0 . 1 0 1 0 3 1}$ \\
\hline
\end{tabular}

Figure 20 (Noor iteration)

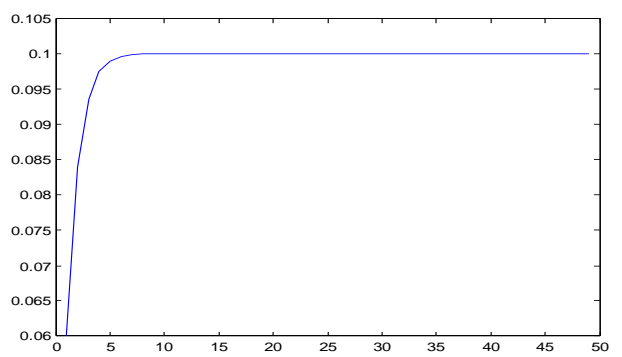

Table 21 ( SP iteration) $s=0.6, s^{\prime}=0.5, s^{\prime \prime}=0.4$

\begin{tabular}{|c|c|}
\hline Number of iterations & $x_{n}$ \\
\hline 1 & 0.088219 \\
\hline 2 & 0.099351 \\
\hline 3 & 0.10081 \\
\hline 4 & 0.101002 \\
\hline 5 & 0.101027 \\
\hline 6 & $\mathbf{0 . 1 0 1 0 3 1}$ \\
\hline 7 & $\mathbf{0 . 1 0 1 0 3 1}$ \\
\hline 8 & $\mathbf{0 . 1 0 1 0 3 1}$ \\
\hline
\end{tabular}

Figure 21 (SP iteration)

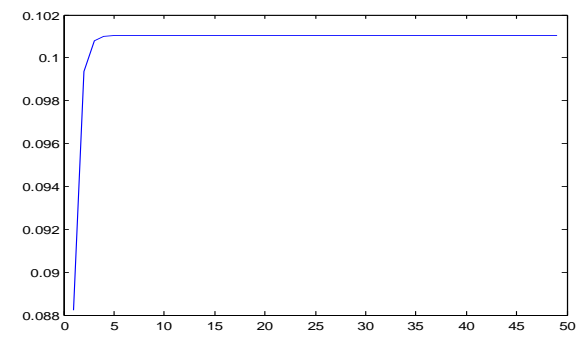

Table 22 (Agarwal et al. iteration ) $s=0.6, s^{\prime}=0.8$

\begin{tabular}{|c|l|}
\hline Number of iterations & $x_{n}$ \\
\hline 1 & 0.100307 \\
\hline 2 & 0.101019 \\
\hline 3 & $\mathbf{0 . 1 0 1 0 3 1}$ \\
\hline 4 & $\mathbf{0 . 1 0 1 0 3 1}$ \\
\hline 5 & $\mathbf{0 . 1 0 1 0 3 1}$ \\
\hline 6 & $\mathbf{0 . 1 0 1 0 3 1}$ \\
\hline
\end{tabular}

Figure 22 (Agarwal et al. iteration )

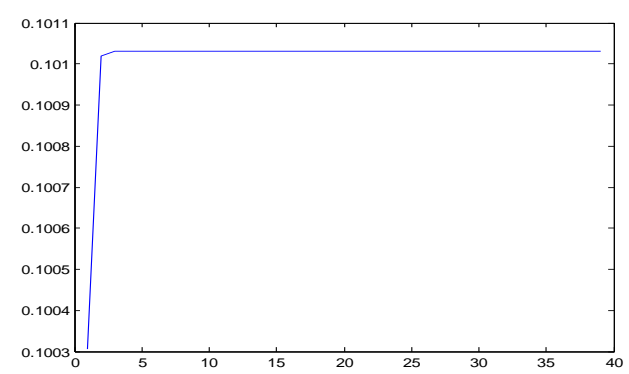

Table 23 (Noor iteration ) $s=0.6, s^{\prime}=0.8, s^{\prime \prime}=0.7$

\begin{tabular}{|c|c|c|c|}
\hline $\begin{array}{c}\text { Num } \\
\text { ber } \\
\text { of } \\
\text { iterat } \\
\text { ions }\end{array}$ & $x_{n}$ & $\begin{array}{c}\text { Number } \\
\text { of } \\
\text { iteration } \\
\mathrm{s}\end{array}$ & $x_{n}$ \\
\hline 1 & 0.06031 & 9 & 0.100997 \\
2 & 0.084601 & 10 & 0.101017 \\
\hline 3 & 0.094399 & 11 & 0.101025 \\
\hline 4 & 0.098353 & 12 & 0.101029 \\
\hline 5 & 0.09995 & 13 & 0.10103 \\
\hline 6 & 0.100595 & 14 & $\mathbf{0 . 1 0 1 0 3 1}$ \\
7 & 0.100855 & 15 & $\mathbf{0 . 1 0 1 0 3 1}$ \\
\hline 8 & 0.10096 & 16 & $\mathbf{0 . 1 0 1 0 3 1}$ \\
\hline
\end{tabular}


Figure 23 (Noor iteration )

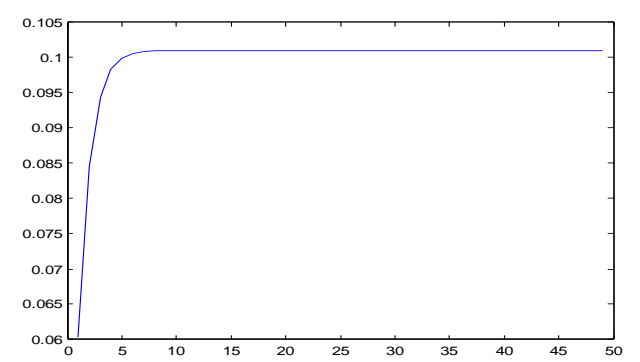

Table 24 (SP iteration ) $s=0.6, s^{\prime}=0.8, s^{\prime \prime}=0.7$

\begin{tabular}{|c|c|}
\hline Number of iterations & $x_{n}$ \\
\hline 1 & 0.098212 \\
\hline 2 & 0.100946 \\
3 & 0.101029 \\
4 & $\mathbf{0 . 1 0 1 0 3 1}$ \\
\hline 5 & $\mathbf{0 . 1 0 1 0 3 1}$ \\
\hline 6 & $\mathbf{0 . 1 0 1 0 3 1}$ \\
\hline
\end{tabular}

Figure 24 (SP iteration)

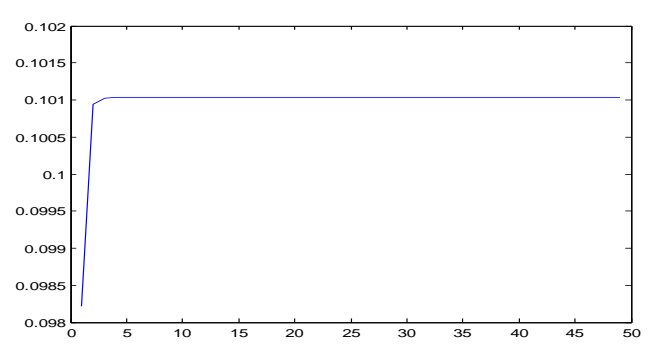

3.3 Fixed points of biquadratic polynomial

Table 25 (Agarwal et al. iteration ) $s=0.6, s^{\prime}=\mathbf{0 . 1}$

\begin{tabular}{|c|c|}
\hline Number of iterations & $x_{n}$ \\
\hline 1 & 0.1 \\
\hline 2 & $\mathbf{0 . 1 0 0 1}$ \\
\hline 3 & $\mathbf{0 . 1 0 0 1}$ \\
\hline 4 & $\mathbf{0 . 1 0 0 1}$ \\
\hline 5 & $\mathbf{0 . 1 0 0 1}$ \\
\hline
\end{tabular}

Figure 25 (Agarwal et al. iteration ) $s=0.6, s^{\prime}=\mathbf{0 . 1}$

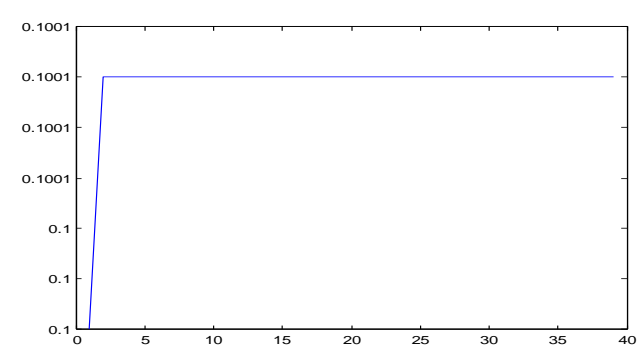

Table 26 (Noor iteration ) $s=0.6, s^{\prime}=s^{\prime \prime}=0.1$

\begin{tabular}{|c|c|c|c|}
\hline $\begin{array}{c}\text { Number } \\
\text { of } \\
\text { iterations }\end{array}$ & $x_{n}$ & $\begin{array}{c}\text { Number } \\
\text { of } \\
\text { iterations }\end{array}$ & $x_{n}$ \\
\hline 1 & 0.06 & 8 & 0.100032 \\
\hline 2 & 0.08401 & 9 & 0.100073 \\
\hline
\end{tabular}

\begin{tabular}{|r|r|r|r|}
\hline 3 & 0.093636 & 10 & 0.100089 \\
\hline 4 & 0.097502 & 11 & 0.100096 \\
\hline 5 & 0.099056 & 12 & 0.100099 \\
\hline 6 & 0.09968 & 13 & $\mathbf{0 . 1 0 0 1}$ \\
\hline 7 & 0.099931 & 14 & $\mathbf{0 . 1 0 0 1}$ \\
\hline
\end{tabular}

Figure 26 (Noor iteration ) $s=0.6, s^{\prime}=s^{\prime \prime}=0.1$

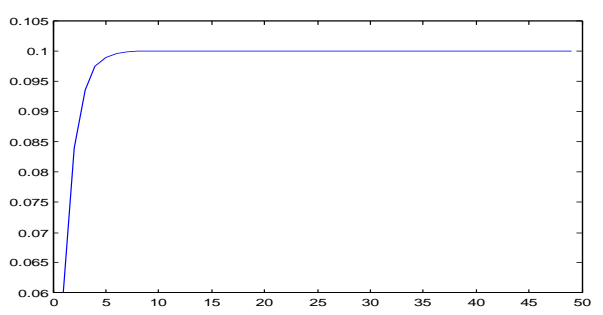

Table $27($ SP iteration $) s=0.6, s^{\prime}=s^{\prime \prime}=\mathbf{0 . 1}$

\begin{tabular}{|c|c|c|c|}
\hline $\begin{array}{c}\text { Number } \\
\text { of } \\
\text { iterations }\end{array}$ & $x_{n}$ & $\begin{array}{c}\text { Number } \\
\text { of } \\
\text { iterations }\end{array}$ & \multicolumn{1}{|c|}{$x_{n}$} \\
\hline 1 & 0.0676 & 7 & 0.100061 \\
\hline 2 & 0.089522 & 8 & 0.100088 \\
\hline 3 & 0.096652 & 9 & 0.100096 \\
\hline 4 & 0.098976 & 10 & 0.100099 \\
\hline 5 & 0.099734 & 11 & $\mathbf{0 . 1 0 0 1}$ \\
\hline 6 & 0.099981 & 12 & $\mathbf{0 . 1 0 0 1}$ \\
\hline
\end{tabular}

Figure 27 (SP iteration ) $s=\mathbf{0 . 6}, s^{\prime}=s^{\prime \prime}=\mathbf{0 . 1}$

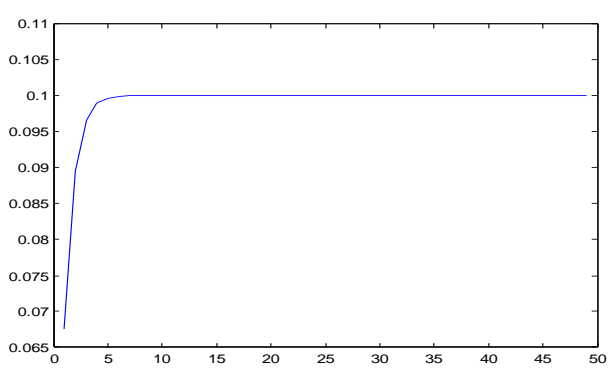

Table 28 (Agarwal et al. iteration ) $s=0.6, s=0.3$

\begin{tabular}{|c|c|}
\hline Number of iterations & $x_{n}$ \\
\hline 1 & 0.1 \\
\hline 2 & $\mathbf{0 . 1 0 0 1}$ \\
\hline 3 & $\mathbf{0 . 1 0 0 1}$ \\
\hline 4 & $\mathbf{0 . 1 0 0 1}$ \\
\hline 5 & $\mathbf{0 . 1 0 0 1}$ \\
\hline
\end{tabular}

Figure 28 (Agarwal et al. iteration ) $s=0.6, s^{\prime}=0.3$ 


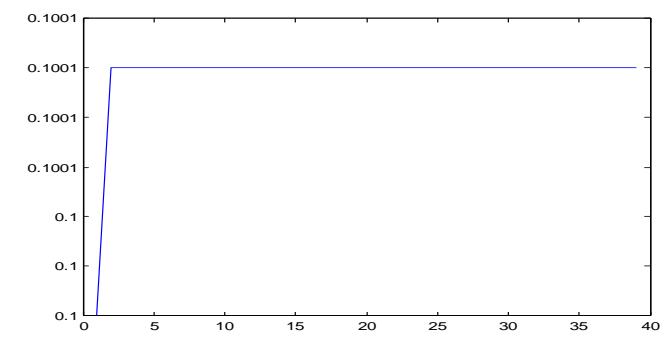

Table 29 (Noor iteration ) $s=0.6, s^{\prime}=0.3, s^{\prime \prime}=0.1$

\begin{tabular}{|c|c|c|c|}
\hline $\begin{array}{c}\text { Number } \\
\text { of } \\
\text { iterations }\end{array}$ & $x_{n}$ & $\begin{array}{c}\text { Number } \\
\text { of } \\
\text { iterations }\end{array}$ & $x_{n}$ \\
\hline 1 & 0.06 & 8 & 0.100033 \\
\hline 2 & 0.084016 & 9 & 0.100073 \\
\hline 3 & 0.093644 & 10 & 0.10009 \\
\hline 4 & 0.097508 & 11 & 0.100096 \\
\hline 5 & 0.099059 & 12 & 0.100099 \\
\hline 6 & 0.099682 & 13 & $\mathbf{0 . 1 0 0 1}$ \\
\hline 7 & 0.099932 & 14 & $\mathbf{0 . 1 0 0 1}$ \\
\hline
\end{tabular}

Figure 29 (Noor Iteration)

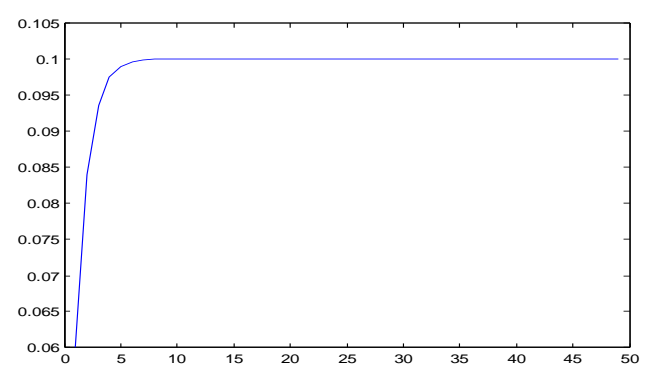

Table 30 (SP iteration ) $s=\mathbf{0 . 6}, s^{\prime}=0.3, s^{\prime \prime}=0.1$

\begin{tabular}{|c|c|c|c|}
\hline $\begin{array}{c}\text { Number } \\
\text { of } \\
\text { iterations }\end{array}$ & $x_{n}$ & $\begin{array}{c}\text { Number } \\
\text { of } \\
\text { iterations }\end{array}$ & $x_{n}$ \\
\hline 1 & 0.074801 & 6 & 0.100074 \\
\hline 2 & 0.093685 & 7 & 0.100094 \\
\hline 3 & 0.098471 & 8 & 0.100099 \\
\hline 4 & 0.099687 & 9 & $\mathbf{0 . 1 0 0 1}$ \\
\hline 5 & 0.099995 & 10 & $\mathbf{0 . 1 0 0 1}$ \\
\hline
\end{tabular}

Figure 30 ( SP Iteration)

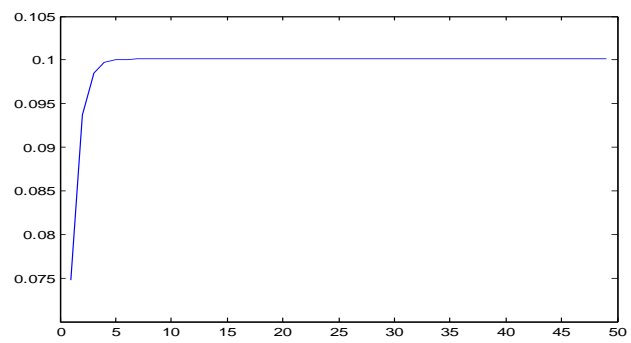

Table 31 (Agarwal et al. iteration ) $s=\mathbf{0 . 6}, s^{\prime}=\mathbf{0 . 5}$

\begin{tabular}{|c|c|}
\hline Number of iterations & $x_{n}$ \\
\hline 1 & 0.100004 \\
\hline 2 & 0.1001 \\
\hline 3 & 0.1001 \\
\hline 4 & 0.1001 \\
\hline 5 & 0.1001 \\
\hline
\end{tabular}

Figure 31 (Agarwal et al. iteration)

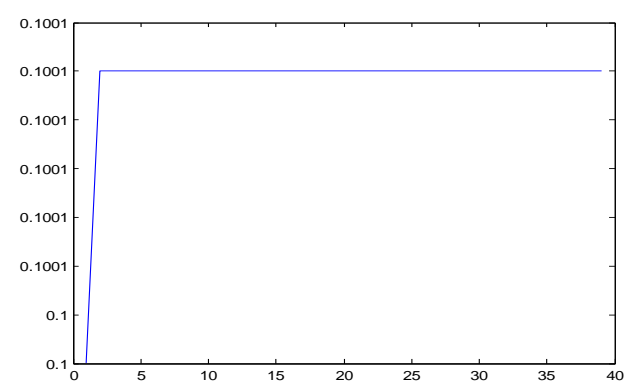

Table 32 (Noor iteration ) $s=0.6, s^{\prime}=0.5, s^{\prime \prime}=0.4$

\begin{tabular}{|c|c|c|c|}
\hline $\begin{array}{c}\text { Number } \\
\text { of } \\
\text { iterations }\end{array}$ & $x_{n}$ & $\begin{array}{c}\text { Number } \\
\text { of } \\
\text { iterations }\end{array}$ & \multicolumn{1}{|c|}{$x_{n}$} \\
\hline 1 & 0.060004 & 8 & 0.100033 \\
\hline 2 & 0.084026 & 9 & 0.100074 \\
\hline 3 & 0.093654 & 10 & 0.10009 \\
\hline 4 & 0.097514 & 11 & 0.100096 \\
\hline 5 & 0.099063 & 12 & 0.100099 \\
\hline 6 & 0.099684 & 13 & $\mathbf{0 . 1 0 0 1}$ \\
\hline 7 & 0.099933 & 14 & $\mathbf{0 . 1 0 0 1}$ \\
\hline
\end{tabular}

Figure 32 ( Noor Iteration)

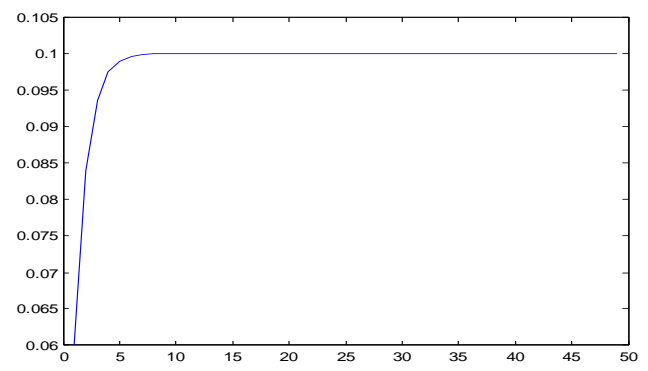

Table 33 (SP iteration ) $s=0.6, s^{\prime}=0.5, s^{\prime \prime}=0.4$

\begin{tabular}{|c|c|}
\hline $\begin{array}{c}\text { Number of } \\
\text { iterations }\end{array}$ & $x_{n}$ \\
\hline 1 & 0.088015 \\
\hline 2 & 0.098633 \\
\hline 3 & 0.099922 \\
\hline 4 & 0.100079 \\
\hline 5 & 0.100098 \\
\hline 6 & $\mathbf{0 . 1 0 0 1}$ \\
\hline 7 & $\mathbf{0 . 1 0 0 1}$ \\
\hline 8 & $\mathbf{0 . 1 0 0 1}$ \\
\hline
\end{tabular}

Figure 33 ( SP Iteration)

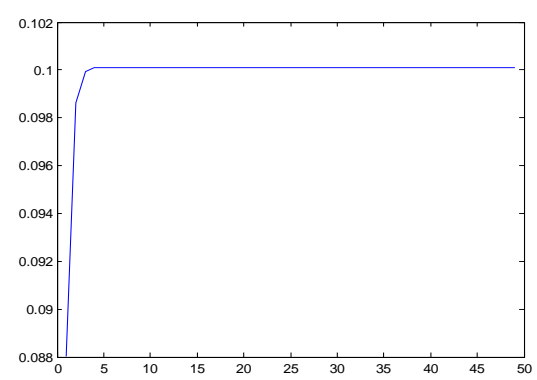

Table 34 (Agarwal et al. iteration ) $s=0.6, s^{\prime}=0.8$ 


\begin{tabular}{|c|c|}
\hline $\begin{array}{c}\text { Number of } \\
\text { iterations }\end{array}$ & $x_{n}$ \\
\hline 1 & 0.100025 \\
\hline 2 & $\mathbf{0 . 1 0 0 1}$ \\
\hline 3 & $\mathbf{0 . 1 0 0 1}$ \\
\hline 4 & $\mathbf{0 . 1 0 0 1}$ \\
\hline 5 & $\mathbf{0 . 1 0 0 1}$ \\
\hline
\end{tabular}

Figure 34 (Agarwal et al. iteration)

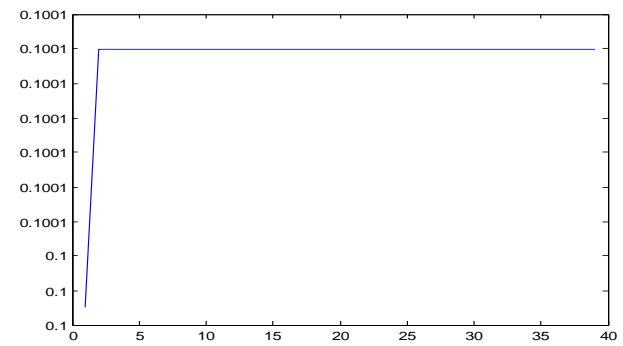

Table 35 (Noor Iteration) $s=\mathbf{0 . 6}, s^{\prime}=\mathbf{0 . 8}, s^{\prime \prime}=0.7$

\begin{tabular}{|c|c|c|c|}
\hline $\begin{array}{c}\text { Number } \\
\text { of } \\
\text { iterations }\end{array}$ & $x_{n}$ & $\begin{array}{c}\text { Number } \\
\text { of } \\
\text { iterations }\end{array}$ & $x_{n}$ \\
\hline 1 & 0.060025 & 8 & 0.100034 \\
\hline 2 & 0.084053 & 9 & 0.100074 \\
\hline 3 & 0.093674 & 10 & 0.10009 \\
\hline 4 & 0.097527 & 11 & 0.100096 \\
\hline 5 & 0.09907 & 12 & 0.100099 \\
\hline 6 & 0.099688 & 13 & $\mathbf{0 . 1 0 0 1}$ \\
\hline 7 & 0.099935 & 14 & $\mathbf{0 . 1 0 0 1}$ \\
\hline
\end{tabular}

Figure 35 (Noor Iteration)

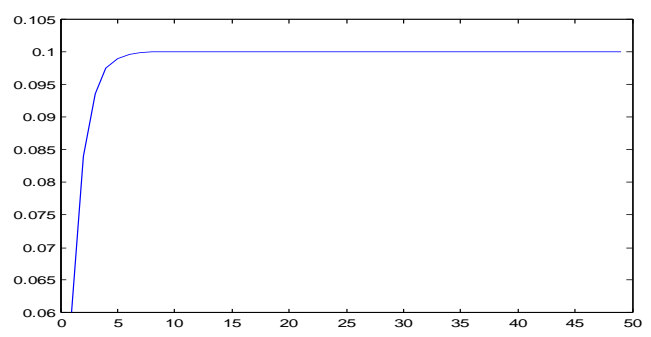

Table 36 (SP Iteration) $s=0.6, s^{\prime}=0.8, s^{\prime \prime}=0.7$

\begin{tabular}{|l|c|}
\hline Number of iterations & $x_{n}$ \\
\hline 1 & 0.097655 \\
\hline 2 & 0.10004 \\
\hline 3 & 0.100099 \\
\hline 4 & $\mathbf{0 . 1 0 0 1}$ \\
\hline 5 & $\mathbf{0 . 1 0 0 1}$ \\
\hline
\end{tabular}

Figure 36 (SP iteration)

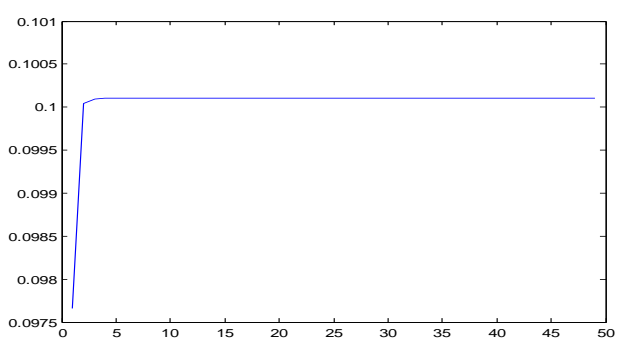

\section{OBSERVATIONS}

From comparative analysis (in the form of tables and graphs) we observe that in case of quadratic polynomial for

(i) $s=0.6, s^{\prime}=0.1, s^{\prime \prime}=0.1$

(ii) $s=0.6, s^{\prime}=0.3, s^{\prime \prime}=0.1$

(iii) $s=0.6, s^{\prime}=0.5, s^{\prime \prime}=0.4$

the decreasing order of convergence rate of iterative schemes is as follows:

Agarwal et al. , SP and Noor iterative scheme.

But for $s=0.6, s^{\prime}=0.8, s^{\prime \prime}=0.7$ the decreasing order of convergence of iterative schemes is as follows:

SP, Agarwal et al. and Noor iterative scheme.

Also in case of cubic and biquadratic polynomial the decreasing order of convergence of iterative schemes is

Agarwal et al., SP and Noor iterative scheme for all above mentioned cases.

\section{CONCLUSION}

Keeping in mind comparative analysis drawn by Rana, Dimri and Tomar[1], Tables 1-36 and observations in section 4 we conclude that

(i) In case of quadratic polynomial for $0<s<1$, $0<s^{\prime}, s^{\prime \prime} \leq \frac{1}{2}$, the decreasing order of convergence of iterative schemes is as follows :

Picard, Agarwal et al., SP, Noor, Ishikawa and Mann iterative scheme.

For $0<s<1,0<s^{\prime}, s^{\prime \prime}>\frac{1}{2}$, Picard and Agarwal et al. iterative schemes shows equivalence while the decreasing order of convergence of iterative schemes is as follows :

SP, Agarwal et al., Mann, Noor and Ishikawa iterative scheme.

(ii) In case of cubic polynomial for $0<s<1,0<s^{\prime}, s^{\prime \prime}<\frac{1}{2}$, the decreasing order of convergence rate of iterative schemes is as follows :

Picard, Agarwal et al., SP, Noor, Ishikawa and Mann iterative scheme.

For $0<s<1,0<s^{\prime}=s^{\prime \prime}=\frac{1}{2}$ Picard and Agarwal et al. schemes shows equivalence while decreasing order of convergence of iterative schemes is as follows :

Agarwal et al., SP, Ishikawa, Noor and Mann iterative scheme.

For $0<s<1,0<s^{\prime}, s^{\prime \prime}>\frac{1}{2}$, the decreasing order of convergence rate of iterative schemes is as follows :

Agarwal et al., SP, Mann, Ishikawa and Noor iterative scheme while Picard and Agarwal et al. schemes shows equivalence.

(iii) In case of biquadratic polynomial for $0<s<1$, $0<s^{\prime}, s^{\prime \prime}<\frac{1}{2}$, Picard and Agarwal et al. schemes shows equivalence and the decreasing order of convergence rate of iterative schemes is as follows :

Agarwal et al., SP, Noor, Ishikawa and Mann iterative scheme

For $0<s<1, s^{\prime}=s^{\prime \prime}=\frac{1}{2}$, Picard and Agarwal et al. iterative schemes shows equivalence and decreasing order of convergence rate of iterative schemes is as follows : 
Agarwal et al., SP, Ishikawa, Noor and Mann iterative scheme.

For $0<s<1,0<s^{\prime}, s^{\prime \prime}>\frac{1}{2}$, the decreasing order of convergence rate of iterative schemes is as follows :

Agarwal et al., SP, Mann, Ishikawa and Noor iterative scheme while Picard and Agarwal et al. schemes shows equivalence. Hence, in case of polynomial functions in complex space, Picard scheme is best for $0<s<1,0<s^{\prime}, s^{\prime \prime} \leq \frac{1}{2}$ while for $0<s$ $<1,0<s^{\prime}, s^{\prime \prime}>\frac{1}{2}$, Agarwal et al. iterative scheme can have better convergence rate than the other iterative schemes.

Futhermore, we conclude that with the increase in power of polynomial, convergence rate of all iterative schemes increase rapidly.

\section{ACKNOWLEDGEMENTS}

The authors would like to thank the referee for his/her valuable comments and suggestions.

\section{REFERENCES}

[1] Rana, R., Dimri, R. C. and Tomar, A. 2011 Remarks on convergence among Picard, Mann and Ishikawa iteration for complex space, volume 21, no 9, May 2011.

[2] Rhoades, B. E. 1976 Comments on two fixed point iteration methods, Journal of Mathematical Analysis and Applications, vol. 56, no. 3, pp. 741-750.

[3] Singh, S. L. 1998 A new approach in numerical praxis, Progress Math.(Varanasi) 32(2), 75-89.
[4] Berinde, V. 2004 Picard iteration converges faster than Mann iteration iteration for a class of quasi-contractive operators, Fixed Point Theory and Applications 2, 97 105.

[5] Berinde, V. 2007 Iterative Approximation of Fixed Points, Editura Efemeride.

[6] Hussian, N., Rafiq, A., D, Bosko and L. Rade 2011 On the rate of convergence of various iterative schemes, Fixed Point Theory and Applications 45,6 pages

[7] Phuengrattana, W., Suantai, S. 2011 On the rate of convergence of Mann, Ishikawa, Noor and SP iterations for continuous functions on an arbitrary interval, Journal of Computational and Applied Mathematics, 235, 3006- 3014

[8] Mann, W. R. 1953 Mean value methods in iteration, Proceedings of the American Mathematical Society, vol.4, pp. 506-510.

[9] Ishikawa, S. 1974 Fixed points by a new iteration method, Proceedings of the American Mathematical Society, vol. 44, no. 1, pp. 147-150.

[10] Agarwal, R.P., O’Regan, D. and Sahu, D.R. 2007 Iterative construction of fixed points of nearly asymptotically nonexpasive mappings, Journal of Nonlinear and Convex Analysis 8(1),61-79.

[11] Noor, M. A. 2000 New approximation schemes for general variational inequalities, Journal of Mathematical Analysis and Applications, vol. 251, no. 1, pp. 217-229. 